\title{
High PD-L1 expression is associated with a favorable prognosis in patients with esophageal squamous cell carcinoma undergoing postoperative adjuvant radiotherapy
}

\author{
CHENXUE JIANG $^{1 *}$, YAOYAO ZHU ${ }^{1 *}$, SHUIQIN TANG ${ }^{2 *}$, GU ZHANG $^{3}$, \\ QINGREN LIN ${ }^{4}$, YAPING XU ${ }^{5}$ and JINBIAO SHANG ${ }^{1}$
}

\author{
${ }^{1}$ First Clinical Medical School, Wenzhou Medical University, Wenzhou, Zhejiang 325035; \\ Departments of ${ }^{2}$ Medical Ethics Committee, ${ }^{3}$ Pathology and ${ }^{4}$ Thoracic Oncology Radiation, \\ Zhejiang Cancer Hospital, Hangzhou, Zhejiang 310022; ${ }^{5}$ Department of Radiation Oncology, \\ Shanghai Pulmonary Hospital, Tongji University School of Medicine, Shanghai 200433, P.R. China
}

Received September 15, 2017; Accepted March 7, 2018

DOI: $10.3892 / \mathrm{ol} .2018 .9747$

\begin{abstract}
Previous results on the prognostic value of programmed death-ligand (PD-L)1 expression in patients with esophageal squamous cell carcinoma (ESCC) remain limited and conflicting. The present study aimed to determine whether PD-L1 expression status predicts prognosis in patients with ESCC, particularly in those undergoing different postoperative treatments. Immunohistochemical staining for PD-L1 was performed on surgical specimens that were obtained from 246 patients with ESCC, who underwent surgical treatment but did not undergo preoperative chemotherapy, radiotherapy, targeted therapy or immune therapy. The association of PD-L1 expression with the clinicopathological factors and the association of PD-L1 expression with survival of patients with ESCC, including subgroups of patients undergoing different postoperative treatments (surgery alone, surgery with adjuvant chemotherapy, surgery with adjuvant radiotherapy and surgery with adjuvant chemo-radiotherapy groups), were statistically analyzed. Positive PD-L1 expression was significantly associated with advanced tumor-node metastasis stage $(\mathrm{P}=0.022)$. Median overall survival (OS) time was compared between patients with positive PD-L1 expression and those with negative PD-L1 expression in the overall patient population. In patients who were treated with postoperative adjuvant radiotherapy, the prognosis was significantly improved in patients who were PD-L1-positive compared with those who were PD-L1-negative $(\mathrm{P}=0.046)$. In
\end{abstract}

Correspondence to: Dr Jinbiao Shang, First Clinical Medical School, Wenzhou Medical University, Road Center North, Wenzhou, Zhejiang 325035, P.R. China

E-mail: shangjb@zjcc.org.cn

${ }^{*}$ Contributed equally

Key words: programmed death-ligand 1, esophageal squamous cell carcinoma, prognosis, radiotherapy patients treated with adjuvant chemotherapy, median OS was poorer in patients with positive PD-L1 expression compared with those with negative PD-L1 expression. However, the difference was not significant. Multivariate Cox regression analysis demonstrated that PD-L1 expression status was not an independent prognostic factor in patients with ESCC. High PD-L1 expression was associated with a favorable prognosis in patients with ESCC undergoing postoperative adjuvant radiotherapy, and it was concluded that patients with positive PD-L1 expression might benefit from postoperative adjuvant radiotherapy.

\section{Introduction}

China has a high incidence of esophageal cancer, as this malignancy had the fourth highest mortality rate and the third highest incidence rate of all malignancies in 2015 (1). Esophageal squamous cell carcinoma (ESCC) is the major histological type of esophageal cancer in China, accounting for $>90 \%$ of all esophageal cancer cases (2). Although the application of combination treatment strategies that consist of surgery, chemotherapy and radiotherapy has improved the prognosis of ESCC, the 5-year overall survival (OS) rate remains low at $20-30 \%$ (3). Therefore, there is an urgent requirement to develop more effective therapeutic and prognostic strategies.

In previous years, notable therapeutic results have been achieved in advanced stages of cancer by blocking immune checkpoint molecules; of which programmed death-1 (PD)-1 and its ligand (PD-L1) have attracted substantial attention (4). Upon binding to PD-1, PD-L1 expressed on tumor cells and tumor-infiltrating lymphocytes (TILs), transduces inhibitory signals to regulate T-lymphocyte activation, tolerance and immune-mediated tissue damage, and promote the immune escape of tumor cells (5-7). Abnormal PD-L1 expression has been associated with the prognosis and therapeutic response in a number of malignancies, including esophageal cancer (8-10). Several studies revealed that PD-L1 positivity was significantly associated with disease progression, response to chemotherapy or radiotherapy, or disease control in ESCC (8-10). Notably, 
PD-L1 expression has been suggested to have positive (8) and negative prediction values in ESCC (9); therefore, the data on the prognostic value of PD-L1 expression in ESCC that is currently available remains limited and conflicting.

The present study aimed to determine the association between PD-L1 expression status and prognosis in an overall population of patients with ESCC and in subgroups of patients with ESCC who received chemotherapy or radiotherapy. The expression of PD-L1 in surgical ESCC specimens from 246 patients was examined, including those who underwent chemotherapy and/or radiotherapy following surgery. The association of PD-L1 expression with clinicopathological characteristics and survival was retrospectively analyzed with the aim of clarifying whether PD-L1 expression status was associated with a favorable or poor prognosis in patients with ESCC, including those undergoing different postoperative treatments.

\section{Patients and methods}

Patients. A total of 246 patients with resectable locally advanced ESCC, who underwent surgical treatment at the Zhejiang Cancer Hospital (Zhejiang, China) between January 2007 and December 2012, were included in the present study. All tissue specimens were obtained from the tissue bank of Zhejiang Cancer Hospital, and all patients provided written informed consent prior to surgery. The Institutional Review Board of Zhejiang Cancer Hospital provided ethical approval. Of the 246 patients, $222(90.2 \%)$ were male and 24 (9.2\%) were female. The median age of these patients at diagnosis was 58.0 years old (range, 37-80 years). The patients had not received preoperative chemotherapy, radiotherapy, targeted therapy or immune therapy. The surgical tumor specimens collected from these patients were used for immunohistochemical staining. Patient characteristics, including age, sex, history of smoking, alcohol drinking, Charlson comorbidity index (CCI) (11) and vessel invasion, and tumor characteristics [size, differentiation, resection margin and TNM stage according to the 7th American Joint Committee on Cancer guidelines (12)] were recorded. Follow-ups were performed using clinical records every three months following patient discharge until mortality or the follow-up deadline (August 2016).

Immunohistochemical staining. Immunohistochemical staining for PD-L1 was manually performed. ESCC specimens obtained from primary tumors were fixed with $10 \%$ formalin at $65^{\circ} \mathrm{C}$, embedded in paraffin and cut into $4-\mu \mathrm{m}$ sections. Tissues were immersed for $10 \mathrm{~min}$ in serial dilutions of xylene for de-waxing (dilution: 100, 90 and 80\%), and then immersed in a graded alcohol series (dilution: 100, 90 and $80 \%$ ) for 10 min to eliminate the xylene. Then, antigen retrieval was performed using PT-Link (Dako; Agilent Technologies, Inc., Santa Clara, CA, USA) subsequent to de-waxing at $95^{\circ} \mathrm{C}$. Following immersion in $3 \%$ hydrogen peroxide to block endogenous peroxidase at room temperature for $30 \mathrm{~min}$, the sections were incubated with an anti-PD-L1 rabbit monoclonal antibody (1:200; cat. no. 13684; E1L3N; Cell Signaling Technology, Inc., Danvers, MA, USA) at $4^{\circ} \mathrm{C}$ overnight, followed by incubation with a horseradish peroxidase-conjugated anti-rabbit secondary antibody (ready to use; cat. no. SM802; Envision FLEX HRP; Dako; Agilent
Table I. Association between PD-L1 expression and clinicopathological factors in patients with esophageal squamous cell carcinoma.

\begin{tabular}{|c|c|c|c|}
\hline \multirow[b]{2}{*}{ Variable } & \multicolumn{2}{|c|}{ PD-L1 } & \multirow[b]{2}{*}{ P-value } \\
\hline & + & - & \\
\hline Age, years & & & 0.312 \\
\hline$<65$ & 48 & 159 & \\
\hline$\geq 65$ & 12 & 27 & \\
\hline Sex, $n$ & & & 0.942 \\
\hline Female & 6 & 18 & \\
\hline Male & 54 & 168 & \\
\hline $\mathrm{CCI}$ & & & 0.600 \\
\hline Low & 8 & 19 & \\
\hline Moderate & 51 & 160 & \\
\hline High & 1 & 7 & \\
\hline Vessel invasion & & & 0.137 \\
\hline Yes & 13 & 59 & \\
\hline No & 47 & 127 & \\
\hline Differentiation & & & 0.542 \\
\hline Well & 17 & 67 & \\
\hline Moderate & 36 & 101 & \\
\hline Poor & 7 & 18 & \\
\hline Tumor location & & & 0.209 \\
\hline Upper & 21 & 77 & \\
\hline Middle & 39 & 103 & \\
\hline Lower & 0 & 6 & \\
\hline $\mathrm{T}$ stage & & & 0.109 \\
\hline $1-2$ & 10 & 136 & \\
\hline $3-4$ & 50 & 50 & \\
\hline N stage & & & 0.213 \\
\hline $0-1$ & 33 & 119 & \\
\hline $2-3$ & 27 & 67 & \\
\hline TMN stage ${ }^{\mathrm{a}}$ & & & 0.022 \\
\hline II & 27 & 132 & \\
\hline III & 33 & 54 & \\
\hline Smoking history & & & 0.837 \\
\hline Yes & 47 & 148 & \\
\hline No & 13 & 38 & \\
\hline Alcohol consumption & & & 0.892 \\
\hline Yes & 46 & 141 & \\
\hline No & 14 & 45 & \\
\hline
\end{tabular}

${ }^{\mathrm{a}}$ According to the 7th American Joint Committee on Cancer guidelines. CCI, Charlson comorbidity index; TNM, tumor-node metastasis; T stage, tumor stage; $\mathrm{N}$ stage, node stage; PD-L1, programmed death-ligand 1.

Technologies, Inc., Santa Clara, CA, USA) for $30 \mathrm{~min}$ at room temperature. Subsequently, horseradish peroxidase activity was detected using 3,3'-diaminobenzidine at room temperature. The sections were counterstained using hematoxylin and examined under a light microscope (magnification, x10 and x40). 
A

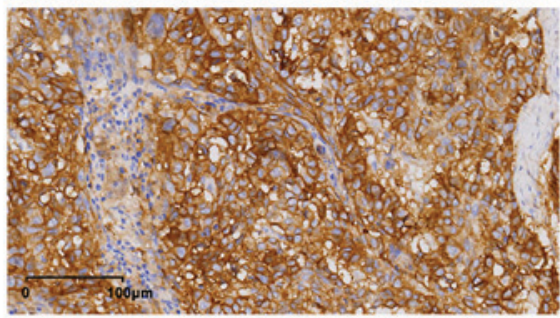

C

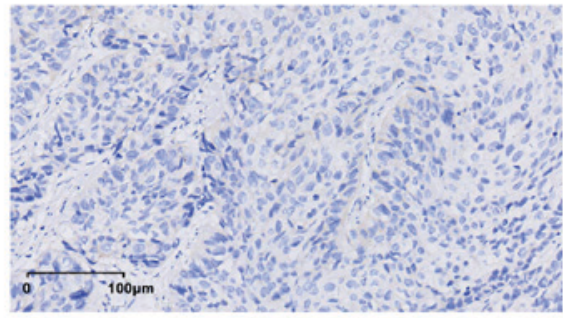

E

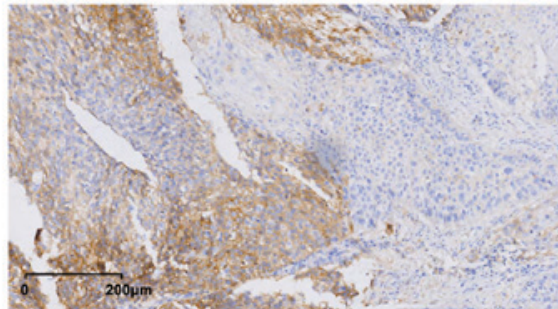

$B$

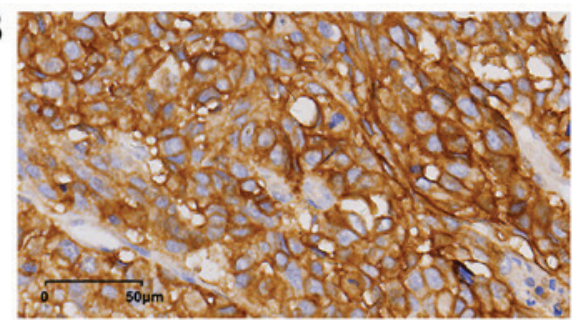

D

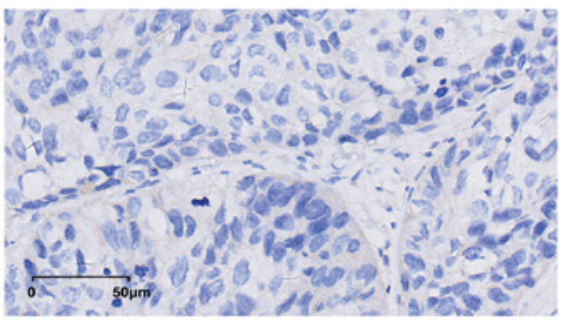

$\mathbf{F}$

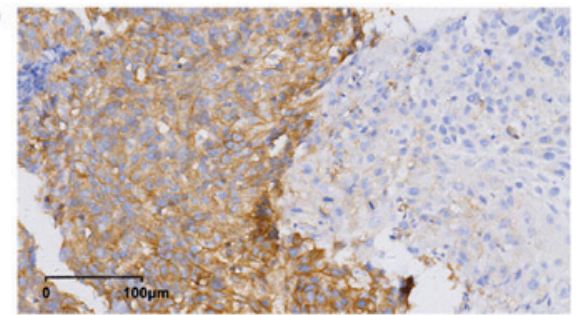

Figure 1. Immunohistochemical staining of PD-L1 in esophageal squamous cell carcinoma tissues. Images obtained from three different patients. Positive expression of PD-L1 [magnification, (A) x20 and (B) x40]. Negative expression of PD-L1 [magnification, (C) x20 and (D) x40]. Heterogeneity of PD-L1 expression [magnification, (E) x10 and (F) x20]. Image B, D and F were magnified from image A, C and E. PD-L1, programmed death-ligand 1.

Evaluation of immunohistochemical staining. Immunoreactivity for PD-L1 was evaluated semi-quantitatively using the $\mathrm{H}$-score, which combines the staining intensity and the percentage of stained tumor cells (13). The staining intensity was graded on a four-point scale: 0 , negative; 1 , weak; 2 , moderate and 3 , strong. The percentage of stained tumor cells was scored between 0 and 100, increasing by $5 \%$ increments. Therefore, the final $\mathrm{H}$-score values obtained ranged from 0 to 300 . The H-scores were separated into low and high categories using the X-tile program (Olympus Corporation, Tokyo, Japan), with the mean H-score as the cutoff value (14). Two experienced pathologists independently calculated the percentage of stained tumor cells and the staining intensity and discussed to reach a consensus in cases where there were disagreements.

Statistical analysis. All statistical analyses were performed using IBM SPSS software (version 22; IBM Corp., Armonk, NY, USA). Associations between clinicopathological variables and PD-L1 expression were analyzed using a Pearson's $\chi^{2}$ test. The Kaplan-Meier method and log rank test were used to calculate the rate of OS and to compare differences between the survival curves. Cox's proportional hazard regression model was utilized to compare categorical variables using backward elimination with a stay level of 0.10 . Logistic regression was performed to investigate the association between PD-L1 expression status and clinicopathological factors using a backward regression procedure that included variables where $\mathrm{P}<0.1$ in the univariate analysis. All P-values were two-tailed, and $\mathrm{P}<0.05$ was considered to indicate a statistically significant difference.

\section{Results}

Expression of PD-L1 in ESCC tissues. PD-L1 was expressed on the membrane and in the cytoplasm, and PD-L1 expression exhibited heterogeneity in the ESCC specimens (Fig. 1). The mean H-score for PD-L1 expression in the patient population was 16 (range, 0-240). According to a cut-off value of 15 obtained using X-tile software analysis, 60 (24.4\%, 60/246) patients were considered positive for PD-L1.

Association between PD-LI expression status and clinicopathological factors in ESCC patients. The clinicopathological characteristics of patients are summarized in Table I. Among these patients, 222 (90.2\%, 222/246) were male and $24(9.8 \%, 24 / 246)$ were female. The median age of these patients was 58.0 years old (range, 37-80 years old) at diagnosis, and the median OS time was 53.0 months [95\% confidence interval (CI), 41.4-64.6 months]. The $\chi^{2}$ test revealed a significant association between positive PD-L1 expression and tumor node metastasis (TNM) stage $(\mathrm{P}=0.022)$. However, there was no association between positive PD-L1 expression and factors, including age, sex, CCI, smoking history, alcohol drinking, vessel invasion, tumor differentiation, tumor location, $\mathrm{T}$ stage and $\mathrm{N}$ stage. Multivariate logistic regression 
analysis also indicated that PD-L1 expression was significantly associated with TNM stage in patients with ESCC ( $\mathrm{P}=0.009$; Table II).

Association between PD-L1 expression and survival in patients with ESCC. Next, the survival outcomes of patients with ESCC based on PD-L1 expression status were assessed. During the follow-up period, $139(56.5 \%, 139 / 246)$ patients succumbed to mortality. The median OS time of the 246 patients was 53.0 months (95\% CI, 41.4-64.6 months). For patients with positive PD-L1 expression, the OS time was 52.4 months (95\% CI, 30.3-74.5 months), whereas for patients with negative PD-L1 expression, the OS time was 56.4 months $(95 \% \mathrm{CI}$, 43.0-69.8 months). However, the difference between the OS time of patients who were positive and those who were negative for PD-L1 was not statistically significant, as presented by the Kaplan-Meier analysis ( $\mathrm{P}=0.466$; Fig. 2).

The survival outcomes in patients receiving different treatments were also assessed (Fig. 3). Among the 246 patients with ESCC, 106 patients received surgical resection alone, 26 patients received surgery in addition to adjuvant chemotherapy, 36 patients received surgery with adjuvant radiotherapy and 78 patients underwent surgery in addition to adjuvant chemoradiotherapy. In the surgery-alone group, there was no significant difference in OS time between PD-L1-positive and -negative patients [41.5 months (95\% CI, 15.7-67.4 months) vs. 58.9 months (95\% CI, 29.2-88.7 months), respectively; P=0.706; Fig. 3A]. In the surgery with adjuvant chemotherapy subgroup, there was also no statistical difference in OS time between PD-L1-positive and -negative patients (21.8 vs. 41.0 months, respectively; $\mathrm{P}=0.765$; Fig. 3B). Notably, in the surgery with adjuvant radiotherapy subgroup, there was a statistical significant difference between patients with positive and negative PD-L1 expression [84.4 months (95\% CI, 55.2-113.6 months) vs. 36.0 months (95\% CI, 18.9-53.1 months), respectively; $\mathrm{P}=0.046$; Fig. 3C]. Furthermore, in the surgery with adjuvant chemoradiotherapy subgroup, there was a tendency for patients with ESCC that had positive PD-L1 expression to have a shorter OS compared with patients with negative PD-L1 expression [38.5 months (95\% CI, 17.8-59.2 months) vs. 69.1 months (95\% CI, 25.2-113.0)]; however, this difference was not significant $(\mathrm{P}=0.061$; Fig. 3D).

Univariate and multivariate analyses of factors affecting survival in patients with ESCC. As presented in Table III, univariate Cox regression analysis revealed that lymph node metastasis and TNM stage were significant prognostic factors for OS in patients with ESCC [ hazard ratio $(\mathrm{HR})=1.61,95 \% \mathrm{CI}$, 1.15-2.26, $\mathrm{P}=0.005$; HR, 1.62, 95\% CI, 1.15-2.29, $\mathrm{P}=0.006$, respectively]. Vessel invasion showed a tendency to be a prognostic factor, but the P-value was not statistically significant ( $\mathrm{HR}=1.47$; 95\% CI, 0.99-2.17; P=0.058). Unexpectedly, PD-L1 expression status was not a significant prognostic factor for OS in patients with ESCC (HR, 1.15, 95\% CI, 0.79-1.68, P=0.467). Multivariate Cox regression hazards analysis revealed that lymph node metastasis $(\mathrm{P}=0.002)$, vessel invasion $(\mathrm{P}=0.011)$ and smoking history $(\mathrm{P}=0.049)$ were independent prognostic factors in patients with ESCC. Patients with advanced N stage, vessel invasion or smoking history had poorer survival than patients with early $\mathrm{N}$ stage, non-vessel invasion of non-smoking history (Table III). Similarly, multivariate analysis indicated
Table II. Multivariate logistic regression analysis of risk factors for programmed death-ligand 1 expression in patients with esophageal squamous cell carcinoma.

\begin{tabular}{lccc}
\hline Variable & OR & $95 \%$ CI & P-value \\
\hline Sex & 1.01 & $0.34-3.01$ & 0.993 \\
Age & 1.33 & $0.61-2.91$ & 0.473 \\
Smoking history & 1.25 & $0.60-2.61$ & 0.553 \\
Alcohol consumption & 1.02 & $0.45-2.33$ & 0.953 \\
Vessel invasion & 2.04 & $1.00-4.17$ & 0.051 \\
T stage & 1.33 & $0.59-3.01$ & 0.494 \\
N stage & 1.82 & $0.57-5.82$ & 0.311 \\
TNM stage & 2.30 & $1.23-4.29$ & 0.009 \\
\hline
\end{tabular}

OR, odds ratio; CI, confidence interval; TNM, tumor-node metastasis; T stage, tumor stage; $\mathrm{N}$ stage, node stage.

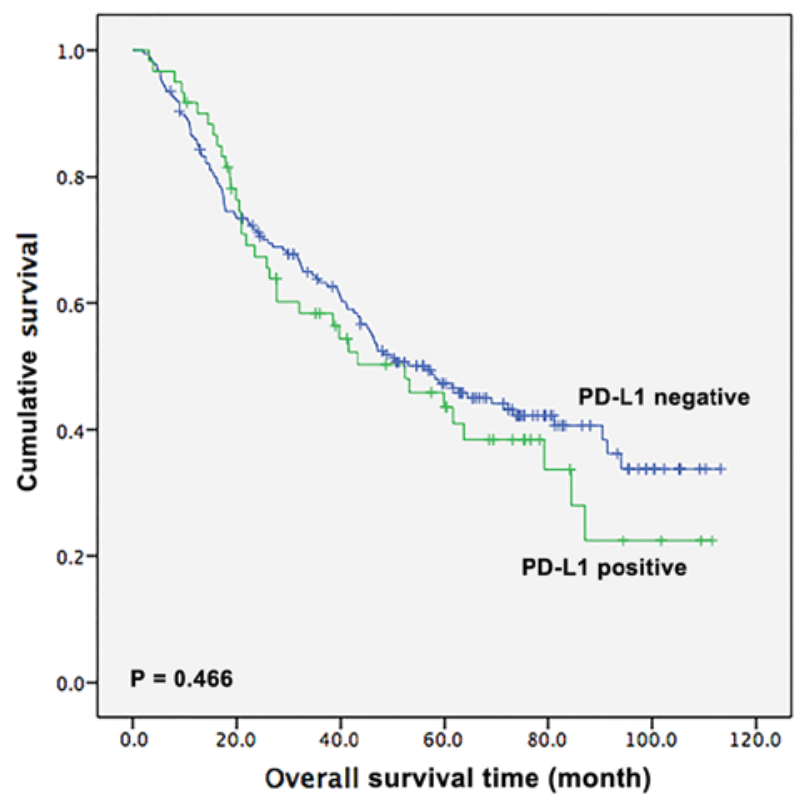

Figure 2. Kaplan-Meier curve of the overall survival time of patients with esophageal squamous cell carcinoma based on PD-L1 expression status. PD-L1, programmed death-ligand 1.

that PD-L1 expression status was not a significant independent prognostic factor (HR, 1.05; 95\% CI, 0.70-1.59; $\mathrm{P}=0.804)$.

\section{Discussion}

The present study investigated the expression of PD-L1 and its association with the clinicopathological factors and clinical outcomes of different treatments in patients with resectable locally advanced ESCC. It was detected that PD-L1 expression was significantly associated with TNM stage in ESCC. High PD-L1 expression is positively associated with advanced TNM stage. In addition, it was revealed that PD-L1 expression was a potential predictor of a favorable response to radiotherapy in patients with ESCC, although median OS time did not differ significantly between patients with positive and negative PD-L1 expression in the overall population. 
Table III. Univariate and multivariate Cox regression analyses of factors affecting the overall survival time of patients with esophageal squamous cell carcinoma $(n=246)$.

A, Univariate analysis

\begin{tabular}{|c|c|c|c|}
\hline Variable & HR & $95 \% \mathrm{CI}$ & P-value \\
\hline
\end{tabular}

PD-L1

Positive vs. negative

$1.152 \quad 0.787-1.684 \quad 0.467$

Sex

Male vs. female

$1.106 \quad 0.624-1.961$

0.730

Age (years)

$\geq 65$ vs. $<65$

$1.080 \quad 0.700-1.665$

0.729

Smoking history

Yes vs. no

$1.388 \quad 0.894-2.156$

0.144

Alcohol drinking

Yes vs. no

$1.100 \quad 0.746-1.621$

0.631

CCI

Low vs. moderate vs. high

$1.176 \quad 0.741-1.866$

0.491

Vessel invasion

Yes vs. no

$1.466 \quad 0.987-2.174$

0.058

Tumor differentiation

Well vs. moderate vs. poor

$1.055 \quad 0.814-1.366$

0.688

Tumor location

Lower vs. middle vs. upper

$1.242 \quad 0.907-1.704$

0.177

T stage

T1-2 vs. T3-4

$1.100 \quad 0.741-1.631$

0.637

$\mathrm{N}$ stage

N0-1 vs. N2-3

$1.613 \quad 1.152-2.257 \quad 0.005$

TNM stage ${ }^{\mathrm{a}}$

II vs. III

$1.623 \quad 1.151-2.288$

0.006

Chemotherapy

Yes vs. no

$1.038 \quad 0.741-1.456$

0.827

Radiotherapy

Yes vs. no

$1.168 \quad 0.838-1.631$

0.359

B, Multivariate analysis

\begin{tabular}{llll}
\hline Variable & HR & $95 \%$ CI & P-value \\
\hline
\end{tabular}

PD-L1

Positive vs. negative

$1.053 \quad 0.698-1.589$

0.804

Sex

Male vs. female

$1.051 \quad 0.545-2.028$

0.882

Age, years

$\geq 65$ vs. $<65$

$1.049 \quad 0.652-1.690$

0.843

Smoking history

Yes vs. no

$1.658 \quad 1.002-2.740$

0.049

Alcohol drinking

Yes vs. no

$1.454 \quad 0.935-2.262$

0.097

CCI

Low vs. moderate vs. high

$1.053 \quad 0.664-1.669$
Table III. Continued.

B, Continued.

\begin{tabular}{lccc}
\hline Variable & HR & $95 \%$ CI & P-value \\
\hline $\begin{array}{l}\text { Vessel invasion } \\
\text { Yes vs. no }\end{array}$ & 1.685 & $1.128-2.518$ & 0.011 \\
$\begin{array}{l}\text { Tumor differentiation } \\
\text { Well vs. moderate vs. poor }\end{array}$ & 1.104 & $0.838-1.456$ & 0.483 \\
$\begin{array}{l}\text { Tumor location } \\
\text { Lower vs. middle vs. upper }\end{array}$ & 1.248 & $0.908-1.718$ & 0.172 \\
$\begin{array}{l}\text { T stage } \\
\text { T1-2 vs. T3-4 }\end{array}$ & 1.068 & $0.665-1.717$ & 0.785 \\
$\begin{array}{l}\text { N stage } \\
\text { N0-1 vs. N2-3 }\end{array}$ & 1.709 & $1.215-2.404$ & 0.002 \\
$\begin{array}{l}\text { TNM stage } \\
\text { II vs. III }\end{array}$ & 1.350 & $0.777-2.347$ & 0.287 \\
$\begin{array}{l}\text { Chemotherapy } \\
\text { Yes vs. no }\end{array}$ & & & \\
$\begin{array}{l}\text { Radiotherapy } \\
\text { Yes vs. no }\end{array}$ & 1.092 & $0.740-1.611$ & 0.659 \\
\hline
\end{tabular}

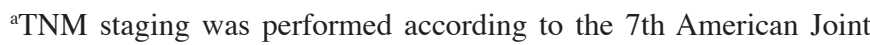
Committee on Cancer guidelines. CCI, Charlson comorbidity index; $\mathrm{HR}$, hazard ratio; CI, confidence interval; PD-L1, programmed death-ligand 1; TNM, tumor-node-metastasis; T stage, tumor stage; $\mathrm{N}$ stage, node stage.

Although one previous study did not reveal any significant associations between PD-L1 expression and clinicopathological factors in 177 patients with ESCC (15), other studies obtained different results. A study conducted by Chen et al (16) on ESCC revealed that PD-L1 expression associated with factors, including upper esophageal location, well-differentiated tumor type, an absence of lymph node metastasis and an early stage of disease suggesting that PD-L1 expression is an indicator of less aggressive tumor types. By contrast, a number of other studies have indicated that basal high PD-L1 expression is positively associated with advanced $\mathrm{T}$ stage, lymph node metastasis and loco-regional failure in ESCC (17-19). In the present study, it was revealed that patients with ESCC with an advanced TNM stage had a significant association with positive PD-L1 expression, and these results suggest that positive PD-L1 expression may be associated with malignant biological behavior in ESCC. However, whether PD-L1 expression results in an advanced TNM stage or whether an advanced TNM stage promotes PD-L1 expression remains unknown. Further studies are required to investigate this.

The present study also revealed that patients with positive and negative PD-L1 expression had similar median OS time (52.4 months vs. 56.4 months, $\mathrm{P}=0.466$ ), which is inconsistent with a majority of previous studies. For instance, three meta-analyses indicated that PD-L1 expression was associated with a poorer OS time in esophageal cancer (20-22). However, one study revealed that a high PD-L1 expression predicted a 
A

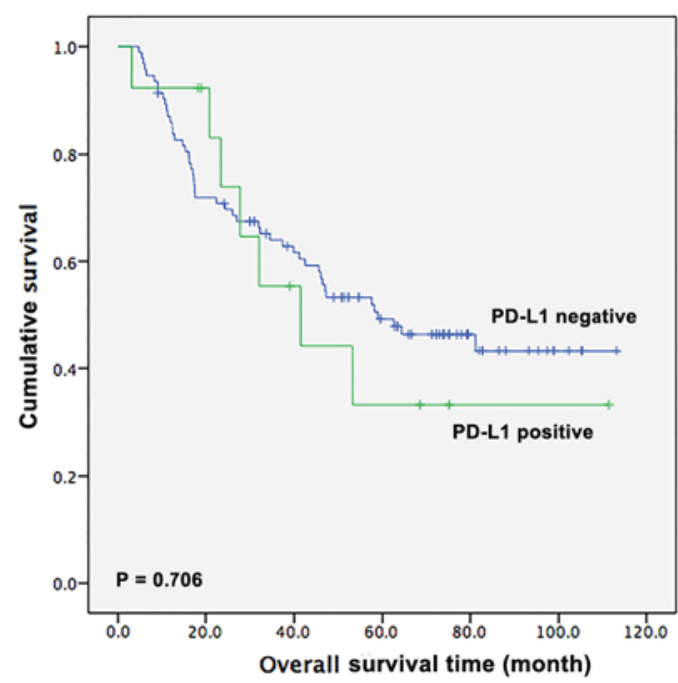

C

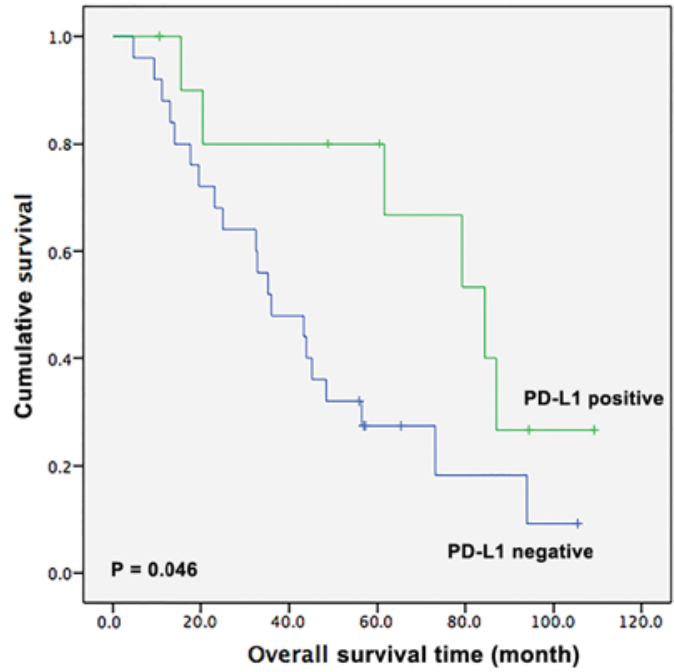

B

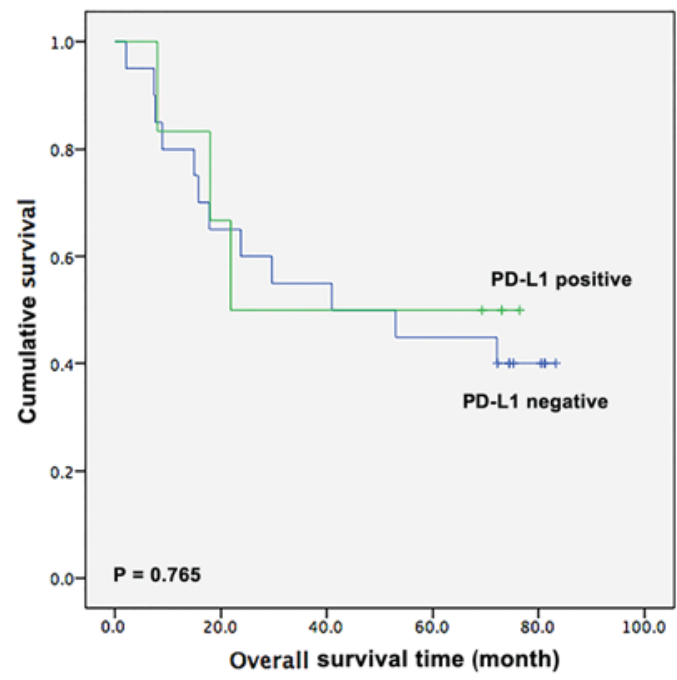

D

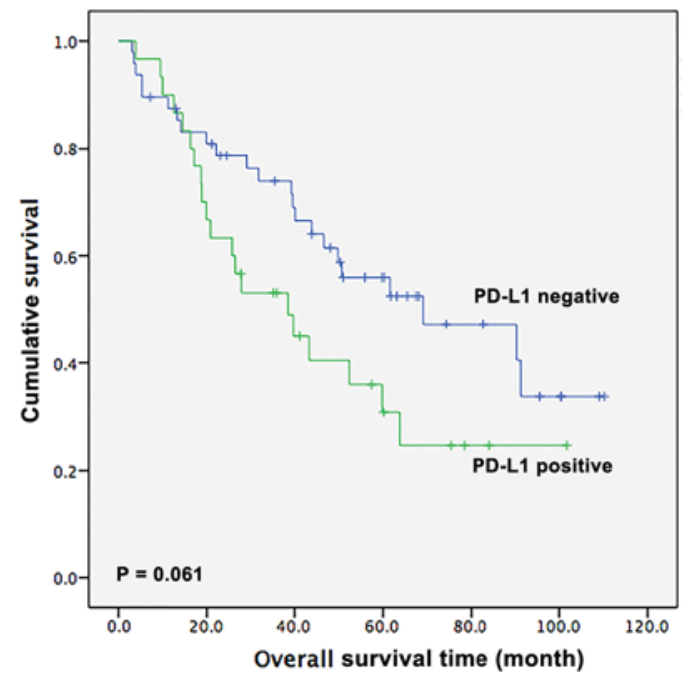

Figure 3. Subgroup analysis of overall survival time in esophageal squamous cell carcinoma patients based on PD-L1 expression. Patients undergoing (A) surgery alone; (B) a combination of surgery and adjuvant chemotherapy; (C) combination of surgery and adjuvant radiotherapy or (D) surgery and adjuvant chemoradiotherapy. PD-L1, programmed death-ligand 1.

favorable prognosis in patients with ESCC (8). The discrepancies among different studies in terms of the association of PD-L1 expression status with clinicopathological factors and survival in ESCC may be due to a combination of several reasons as follows: Different sensitivities of antibodies used; different cut-off values for positive PD-L1 staining; non-uniform PD-L1 expression (as detected in Fig. 1E and F in the present study) and different sampling timing and location (23). In the present study, no association was detected between PD-L1 expression and prognosis in ESC. Future studies should carefully address these issues in order to standardize the immunohistochemical staining procedure and interpretation of immunohistochemical results, enabling the comparison of results obtained from different studies.

Notably, the results of the present study indicated that in patients treated with adjuvant radiotherapy, the prognosis was significantly improved in patients with positive PD-L1 expression compared with those with negative PD-L1 expression (median OS time, 84.4 months vs. 36.0 months; $\mathrm{P}=0.046$ ). In patients treated with adjuvant chemotherapy, median OS was poorer in PD-L1-positive patients compared with
PD-L1-negative patients, although there was no significant statistical difference (21.8 months vs. 41.0 months, $\mathrm{P}=0.765$ ). In agreement with this finding, a number of previous studies have indicated that there is an association between PD-L1 expression and the outcomes of different types of clinical management. In a study of 177 Japanese patients with ESCC who underwent an esophagectomy without preoperative therapy, PD-L1 expression was significantly associated with an improved prognosis in patients who underwent surgery alone, but not in patients treated with surgery plus postoperative adjuvant chemotherapy (15). Another study of 180 Japanese patients with ESCC who were treated by radical resection with or without preoperative neoadjuvant chemotherapy revealed that PD-L1 expression correlated with a poorer OS time in patients treated with preoperative neoadjuvant chemotherapy, but not in those without preoperative neoadjuvant chemotherapy (24). Theoretically, ESCC is moderately sensitive to chemotherapy, but the role of postoperative chemotherapy against ESCC has been debated. The National Comprehensive Cancer Network only recommends postoperative chemotherapy for patients with ESCC with R1 (cancer cells present by microscopy at 
the resection) or $\mathrm{R} 2$ resections (tumor tissue present by naked eyes at the resection margin) (25). The present study revealed that PD-L1-positive patients with ESCC do not benefit from postoperative adjuvant chemotherapy, which indicates that chemotherapy not only has a direct cytotoxic effect on tumor cells, but may also affect the tumor immune system (15).

Radiotherapy is one of the main types of treatment for ESCC (25). Radiation increases the leakage of tumor antigens by killing tumor cells and promotes the activation of tumor-specific $\mathrm{T}$ cells by increasing the ability of antigen-presenting cells to display antigens on their surface (26). Previous studies have documented the synergism between several types of immunotherapy and radiotherapy (27). Previous preclinical studies have revealed that PD-L1 expression may be upregulated in tumor cells by radiation therapy $(28,29)$. In addition, anti-PD-L1 therapy combined with radiotherapy improves survival compared with radiotherapy alone in mice (30).

PD-L1 expression on tumor cells is generally considered to be one of the mechanisms for immune evasion via downregulating the function of TILs $\left(\mathrm{CD}^{+} \mathrm{T}-1 \mathrm{ymphocytes}\right.$ and CD4 ${ }^{+}$T-lymphocytes) or activating the epidermal growth factor receptor signaling pathway (31-33). However, in the present study, positive PD-L1 expression was associated with a favorable survival in patients who underwent radiotherapy. It was hypothesized that patients with PD-L1 expression had highly immunogenic tumors prior to radiation therapy, which indicates a strong adaptive immune response. In patients with PD-L1 expression, there was an increased infiltration of other subpopulations of TILs, which has been reported to be a strong predictor of good prognosis for patients with ESCC (34). A number of studies reported that high PD-L1 expression on tumor cells was associated with increased intraepithelial CD $3^{+}$ T-lymphocytes, and both factors were associated with a favorable prognosis in ESCC $(8,35)$.

Autophagy is an evolutionarily conserved process for the clearance of damaged or superfluous intracellular products via the lysosomal-mediated pathway (36). Autophagy is associated with a decrease in radiosensitivity. Autophagy inhibitor combined with radiotherapy resulted in enhanced cytotoxicity of radiotherapy in ESCC cells by decreasing the expression of autophagy-associated gene Beclin1 and arresting the cell cycle at the $\mathrm{G}_{2} / \mathrm{M}$ phase $(37,38)$. Tumor-intrinsic PD-L1 signals promote mammalian target of rapamycin (MTORC)1 and inhibit MTORC2, therefore inhibiting autophagy and enhancing the sensitivity to growth suppression by autophagy inhibitors (39). However, a number of studies reported that PD-L1 promoted immune evasion via activation of the protein kinase B/mTOR oncogenic pathway $(40,41)$. Other studies reported that PD-L1 expression was associated with resistance to anticancer therapies, including radiotherapy and chemotherapy $(19,42)$.

The mechanisms responsible for the survival benefit of PD-L1 in patients with ESCC receiving radiotherapy remain to be further investigated. The present study was based on surgical samples obtained from patients with ESCC who did not undergo preoperative chemotherapy, radiotherapy or immunotherapy, suggesting that basal PD-L1 expression status may serve as a potential predictive factor of the effects of adjuvant radiotherapy. Additional research should be performed to investigate the potential molecular mechanism of these associations for ESCC. Studies with larger sample sizes are required to clarify this issue.

The present study was subjected to certain limitations. First, although the present study had a larger sample size compared with other studies $(16,43)$, there was still bias due to its retrospective design. Secondly, only $24.4 \%$ of these patients were regarded as PD-L1-positive, while a meta-analysis demonstrated that nearly $50 \%$ of patients with gastrointestinal cancer were positive for PD-L1 expression, regardless of the evaluation method used (20). The standard used for the positive expression of PD-L1 in the present study was inconsistent with several previous studies (20-22), and there was no unified standard for the positive expression of PD-L1. Whether the threshold selected to determine PD-L1 expression was positive or negative may be arbitrary. In the present study, PD-L1 expression in a number of ESCC tumor tissues appeared to be heterogeneous (Fig. 1E and F). Consistent with this observation, previous studies have revealed that PD-L1 expression was heterogeneous in other malignant tumors (44-46). Therefore, heterogeneous expression may be another reason why the PD-L1 positive rate in the present study was lower compared with those in other studies (20-22). Finally, as no standardized PD-L1 immunohistochemistry assay is currently available, caution should be taken in the interpretation of these results.

In conclusion, the present study demonstrates, to the best of our knowledge, for the first time that high PD-L1 expression in associated with a favorable prognosis in patients with ESCC undergoing postoperative adjuvant radiotherapy. However, this association was not consistent in the overall ESCC population. Therefore, patients with positive PD-L1 expression may benefit from postoperative adjuvant radiotherapy. However, further studies are required in order to confirm these results.

\section{Acknowledgements}

The present study was presented as a conference abstract (Oral no. 117) on 2-5 September, 2017 at the 14th World Conference Global Perspectives in Esophageal Diseases in Geneva, Switzerland.

\section{Funding}

Not applicable.

\section{Availability of data and materials}

All data generated or analyzed during this study are included in this published article.

\section{Authors' contributions}

YX, JS and GZ contributed to the conception of the study and method selection. CJ, YZ, ST and QL made substantial contribution to the acquisition and arrangement of data. CJ, YZ, ZG and QL analyzed the data. CJ, YZ and ST drafted the manuscript. All authors agreed to be accountable for all aspects of the work and to ensure that questions related to the accuracy and integrity of any part of the work are appropriately investigated and resolved. All authors gave final approval of the version to be published. 


\section{Ethics approval and consent to participate}

All tissue specimens were obtained from the tissue bank of Zhejiang Cancer Hospital, and all patients provided written informed consent prior to surgery. The present study was approved by the Medical Ethics Committee of Zhejiang Cancer Hospital ethically (Ethics Approval document IRB-2017-195).

\section{Patient consent for publication}

The patients of our study provided written informed consent for the publication of any associated data and accompanying images.

\section{Competing interests}

The authors declare that there are no competing interests.

\section{References}

1. Chen W, Zheng R, Baade PD, Zhang S, Zeng H, Bray F, Jemal A, $\mathrm{Yu}$ XQ and He J: Cancer statistics in China, 2015. CA Cancer J Clin 66: 115-132, 2016.

2. Torre LA, Bray F, Siegel RL, Ferlay J, Lortet-Tieulent J and Jemal A: Global cancer statistics, 2012. CA Cancer J Clin 65: 87-108, 2015.

3. Tepper J, Krasna MJ, Niedzwiecki D, Hollis D, Reed CE, Goldberg R, Kiel K, Willett C, Sugarbaker D and Mayer R: Phase III trial of trimodality therapy with cisplatin, fluorouracil, radiotherapy, and surgery compared with surgery alone for esophageal cancer: CALGB 9781. J Clin Oncol 26: 1086-1092, 2008.

4. Nishino M, Ramaiya NH, Hatabu H and Hodi FS: Monitoring immune-checkpoint blockade: Response evaluation and biomarker development. Nat Rev Clin Oncol 14: 655-688, 2017.

5. Sharpe AH and Freeman GJ: The B7-CD28 superfamily. Nat Rev Immunol 2: 116-126, 2002.

6. Francisco LM, Sage PT and Sharpe AH: The PD-1 pathway in tolerance and autoimmunity. Immunol Rev 236: 219-242, 2010.

7. Ceeraz S, Nowak EC and Noelle RJ: B7 family checkpoint regulators in immune regulation and disease. Trends Immunol 34 $556-563,2013$

8. Jesinghaus M, Steiger K, Slotta-Huspenina J, Drecoll E, Pfarr N, Meyer P, Konukiewitz B, Bettstetter M, Wieczorek K, Ott K, et al: Increased intraepithelial CD3+ T-lymphocytes and high PD-L1 expression on tumor cells are associated with a favorable prognosis in esophageal squamous cell carcinoma and allow prognostic immunogenic subgrouping. Oncotarget 8: 46756-46768, 2017

9. Jiang Y, Lo AWI, Wong A, Chen W, Wang Y, Lin L and Xu J: Prognostic significance of tumor-infiltrating immune cells and PD-L1 expression in esophageal squamous cell carcinoma. Oncotarget 8: 30175-30189, 2017.

10. Zhang W, Pang Q, Zhang X, Yan C, Wang O, Yang J, Yu S, Liu X, Pan Y, Yuan Z, et al: Programmed death-ligand 1 is prognostic factor in esophageal squamous cell carcinoma and is associated with epidermal growth factor receptor. Cancer Sci 108: 590-597, 2017

11. Yamashita K, Watanabe M, Mine S, Fukudome I, Okamura A, Yuda M, Hayami M and Imamura Y: The impact of the Charlson comorbidity index on the prognosis of esophageal cancer patients who underwent esophagectomy with curative intent. Surg Today 48: 632-639, 2018.

12. Edge SB and Compton CC: The American Joint Committee on Cancer: The 7th edition of the AJCC cancer staging manual and the future of TNM. Ann Surg Oncol 17: 1471-1474, 2010.

13. Chen LJ, Sun J, Wu HY, Zhou SM, Tan Y, Tan M, Shan BE, Lu BF and Zhang XG: B7-H4 expression associates with cancer progression and predicts patient's survival in human esophageal squamous cell carcinoma. Cancer Immunol Immunother 60: 1047-1055, 2011

14. Camp RL, Dolled-Filhart M and Rimm DL: X-tile: A new bio-informatics tool for biomarker assessment and outcome-based cut-point optimization. Clin Cancer Res 10: 7252-7259, 2004.

15. Wakita A, Motoyama S, Nanjo H, Sato Y, Yoshino K, Sasaki T, Kawakita Y, Liu J, Imai K, Saito H and Minamiya Y: PD-L1 expression is a prognostic factor in patients with thoracic esophageal cancer treated without adjuvant chemotherapy. Anticancer Res 37: 1433-1441, 2017.
16. Chen K, Cheng G, Zhang F, Zhang N, Li D, Jin J, Wu J, Ying L, Mao $W$ and Su D: Prognostic significance of programmed death-1 and programmed death-ligand 1 expression in patients with esophageal squamous cell carcinoma. Oncotarget 7: 30772-30780, 2016.

17. Loos M, Langer R, Schuster T, Gertler R, Walch A, Rauser S, Friess $\mathrm{H}$ and Feith M: Clinical significance of the costimulatory molecule B7-H1 in Barrett carcinoma. Ann Thorac Surg 91: 1025-1031, 2011.

18. Chen L, Deng H, Lu M, Xu B, Wang Q, Jiang J and Wu C: B7-H1 expression associates with tumor invasion and predicts patient's survival in human esophageal cancer. Int J Clin Exp Pathol 7: 6015-6023, 2014

19. Chen MF, Chen PT, Chen WC, Lu MS, Lin PY and Lee KD: The role of PD-L1 in the radiation response and prognosis for esophageal squamous cell carcinoma related to IL-6 and T-cell immunosuppression. Oncotarget 7: 7913-7924, 2016.

20. Huang B, Chen L, Bao C, Sun C, Li J, Wang L and Zhang X: The expression status and prognostic significance of programmed cell death 1 ligand 1 in gastrointestinal tract cancer: A systematic review and meta-analysis. Onco Targets Ther 8: 2617-2625, 2015.

21. Wu P, Wu D, Li L, Chai Y and Huang J: PD-L1 and survival in solid tumors: A meta-analysis. PLoS One 10: e0131403, 2015

22. Pyo JS, Kang G and Kim JY: Prognostic role of PD-L1 in malignant solid tumors: A meta-analysis. Int J Biol Markers 32: e68-e74, 2017.

23. Wang X, Teng F, Kong L and Yu J: PD-L1 expression in human cancers and its association with clinical outcomes. Onco Targets Ther 9: 5023-5039, 2016.

24. Tanaka K, Miyata H, Sugimura K, Kanemura T, Hamada-Uematsu M, Mizote Y, Yamasaki M, Wada H, Nakajima K, Takiguchi S, et al: Negative influence of programmed death-1-ligands on the survival of esophageal cancer patients treated with chemotherapy. Cancer Sci 107: 726-733, 2016.

25. National Comprehensive Cancer Network (NCCN). Clinical Practice Guidelines in Oncology. Esophageal and Esophagogastric Junction Cancers, Version 2.2017. 2017.

26. Lim SH, Hong M, Ahn S, Choi YL, Kim KM, Oh D, Ahn YC, Jung SH, Ahn MJ, Park K, et al: Changes in tumour expression of programmed death-ligand 1 after neoadjuvant concurrent chemoradiotherapy in patients with squamous oesophageal cancer. Eur J Cancer 52: 1-9, 2016.

27. Formenti SC and Demaria S: Combining radiotherapy and cancer immunotherapy: A paradigm shift. J Natl Cancer Inst 105: 256-265, 2013

28. Dovedi SJ, Adlard AL, Lipowska-Bhalla G, McKenna C, Jones S, Cheadle EJ, Stratford IJ, Poon E, Morrow M, Stewart R, et al: Acquired resistance to fractionated radiotherapy can be overcome by concurrent PD-L1 blockade. Cancer Res 74: 5458-5468, 2014.

29. Kelly RJ, Zaidi AH, Smith MA, Omstead AN, Kosovec JE, Matsui D, Martin SA, DiCarlo C, Werts ED, Silverman JF, et al: The dynamic and transient immune microenvironment in locally advanced esophageal adenocarcinoma post chemoradiation. Ann Surg, 2017.

30. Zeng J, See AP, Phallen J, Jackson CM, Belcaid Z, Ruzevick J, Durham N, Meyer C, Harris TJ, Albesiano E, et al: Anti-PD-1 blockade and stereotactic radiation produce long-term survival in mice with intracranial gliomas. Int J Radiat Oncol Biol Phys 86: 343-349, 2013.

31. Leng C, Li Y, Qin J, Ma J, Liu X, Cui Y, Sun H, Wang Z, Hua X, $\mathrm{Yu}$ Y, et al: Relationship between expression of PD-L1 and PD-L2 on esophageal squamous cell carcinoma and the antitumor effects of CD8+ T cells. Oncol Rep 35: 699-708, 2016.

32. Chen K, Zhu Z, Zhang N, Cheng G, Zhang F, Jin J, Wu J, Ying L, Mao W and Su D: Tumor-infiltrating CD4+ lymphocytes predict a favorable survival in patients with operable esophageal squamous cell carcinoma. Med Sci Monit 23: 4619-4632, 2017.

33. Zhang W, Pang Q, Yan C, Wang Q, Yang J, Yu S, Liu X, Yuan Z, Wang P and Xiao Z: Induction of PD-L1 expression by epidermal growth factor receptor-mediated signaling in esophageal squamous cell carcinoma. Onco Targets Ther 10: 763-771, 2017.

34. Sudo T, Nishida R, Kawahara A, Saisho K, Mimori K, Yamada A, Mizoguchi A, Kadoya K, Matono S, Mori N, et al: Clinical impact of tumor-infiltrating lymphocytes in esophageal squamous cell carcinoma. Ann Surg Oncol 24: 3763-3770, 2017.

35. Li J, Tang Y, Huang L, Yu Q, Hu G, Zou Y and Yuan X: A high number of stromal tumor-infiltrating lymphocytes is a favorable independent prognostic factor in M0 (stages I-III) esophageal squamous cell carcinoma. Dis Esophagus 30: 1-7, 2016. 
36. Chiu B, Jantuan E, Shen F, Chiu B and Sergi C: Autophagy-inflammasome interplay in heart failure: A systematic review on basics, pathways, and therapeutic perspectives. Ann Clin Lab Sci 47: 243-252, 2017.

37. Apel A, Herr I, Schwarz H, Rodemann HP and Mayer A: Blocked autophagy sensitizes resistant carcinoma cells to radiation therapy. Cancer Res 68: 1485-1494, 2008.

38. Chen Y, Song H, Lu Y, Li X, Chen T, Zhang Y, Xue JX, Liu H, Kan B, Yang $\mathrm{G}$ and $\mathrm{Fu} \mathrm{T}$ : Autophagy inhibition contributes to radiation sensitization of esophageal squamous carcinoma cells. Dis Esophagus 24: 437-443, 2011.

39. Clark CA, Gupta HB and Curiel TJ: Tumor cell-intrinsic CD274/PD-L1: A novel metabolic balancing act with clinical potential. Autophagy 13: 987-988, 2017.

40. Parsa AT, Waldron JS, Panner A, Crane CA, Parney IF, Barry JJ, Cachola KE, Murray JC, Tihan T, Jensen MC, et al: Loss of tumor suppressor PTEN function increases B7-H1 expression and immunoresistance in glioma. Nat Med 13: 84-88, 2007.

41. Dong L, Lv H, Li W, Song Z, Li L, Zhou S, Qiu L, Qian Z, Liu X, Feng L, et al: Co-expression of PD-L1 and p-AKT is associated with poor prognosis in diffuse large B-cell lymphoma via PD-1/PD-L1 axis activating intracellular AKT/mTOR pathway in tumor cells. Oncotarget 7: 33350-33362, 2016.

42. Afreen S and Dermime S: The immunoinhibitory B7-H1 molecule as a potential target in cancer: Killing many birds with one stone. Hematol Oncol Stem Cell Ther 7: 1-17, 2014.
43. Hatogai K, Fujii S, Kojima T, Daiko H, Nomura S, Doi T, Kitano S, Ohtsu A, Takiguchi Y, Yoshino T and Ochiai A: Large-scale comprehensive immunohistochemical biomarker analyses in esophageal squamous cell carcinoma. J Cancer Res Clin Oncol 143: 2351-2361, 2017.

44. Madore J, Vilain RE, Menzies AM, Kakavand H, Wilmott JS, Hyman J, Yearley JH, Kefford RF, Thompson JF, Long GV, et al: PD-L1 expression in melanoma shows marked heterogeneity within and between patients: Implications for anti-PD-1/PD-L1 clinical trials. Pigment Cell Melanoma Res 28: 245-253, 2015.

45. McLaughlin J, Han G, Schalper KA, Carvajal-Hausdorf D, Pelekanou V, Rehman J, Velcheti V, Herbst R, LoRusso P and Rimm DL: Quantitative assessment of the heterogeneity of PD-L1 expression in non-small-cell lung cancer. JAMA Oncol 2: 46-54, 2016.

46. Rehman JA, Han G, Carvajal-Hausdorf DE, Wasserman BE, Pelekanou V, Mani NL, McLaughlin J, Schalper KA and Rimm DL: Quantitative and pathologist-read comparison of the heterogeneity of programmed death-ligand 1 (PD-L1) expression in non-small cell lung cancer. Modern Pathol 30: 340-349, 2017.

This work is licensed under a Creative Commons Attribution-NonCommercial-NoDerivatives 4.0 International (CC BY-NC-ND 4.0) License. 